\title{
Sobre a existência de soluções para equações integrais de Volterra em escalas temporais
}

\section{On the existence of solutions for Volterra integral equations on time scales}

Iguer Luis Domini dos Santos

Universidade Estadual Paulista (UNESP), Departamento de Matemática

Ilha Solteira, SP, Brasil

iguer.santos@unesp.br

\section{Informações do Artigo}

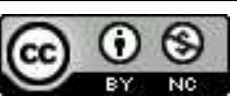

Histórico do Artigo

Submissão: 11 de junho de 2018.

Aceite: 07 de dezembro de 2018.

\section{Palavras-chave}

Equações integrais de Volterra

Existência de soluções

Escalas temporais

\section{Resumo}

Neste trabalho, estudamos a existência de soluções para equações integrais de Volterra em escalas temporais. Utilizando o Teorema do Ponto Fixo de Schäfer, estabelecemos um resultado de existência de soluções para equações integrais de Volterra em escalas temporais. O resultado obtido aqui se soma aos resultados considerados na literatura.

\section{Keywords}

Volterra integral equations

Existence of solutions

Time scales

\begin{abstract}
In this work, we study the existence of solutions for Volterra integral equations on time scales. Using the Schäfer's Fixed Point Theorem, we have established a result of the existence of solutions for Volterra integral equations on time scales. The result obtained here joins the results considered in the literature.
\end{abstract}

\section{Introdução}

A teoria de escalas temporais foi introduzida por Hilger (1988) em sua tese para unificar o cálculo de diferença e o cálculo diferencial. Dessa forma, as equações dinâmicas em escalas temporais unificam o estudo das equações diferenciais ordinárias e das equações de diferenças. Desde a introdução do cálculo em escalas temporais por Hilger (1988), uma vasta literatura tem sido produzida na teoria de escalas temporais. Assim, equações dinâmicas em escalas temporais foram estudadas, por exemplo, em Bohner e Peterson (2001), Bohner e Peterson (2003) e Santos (2015). Já as inclusões dinâmicas em escalas temporais, uma generalização das equações dinâmicas em escalas temporais, foram abordadas, por exemplo, em Atici e Biles (2004), Frigon e Gilbert (2011) e Santos e Silva (2013). Quanto ao estudo de equações integrais de Volterra em escalas temporais, 
objeto do presente trabalho, este pode ser encontrado em Karpuz (2014), Messina e Vecchio (2015), Messina et al. (2015), Santos (2015) e Kulik e Tisdell (2008). A existência e unicidade de soluções para equações integrais de Volterra generalizadas em escalas temporais é estudada em Karpuz (2014). Já a estabilidade de soluções para equações integrais de Volterra em escalas temporais é estudada por Messina e Vecchio (2015) e por Messina et al. (2015). Por sua vez, Santos (2015) estuda propriedades de soluções para equações integrais de Volterra em escalas temporais. Por fim, em Kulik e Tisdell (2008) são introduzidas a teoria qualitativa básica e a teoria quantitativa básica de equações integrais de Volterra em escalas temporais.

Aqui, nós estudamos a seguinte classe de equações integrais de Volterra em escalas temporais

$$
x(t)=f(t)+\int_{[a, t)_{\mathbb{T}}} k(t, s, x(\sigma(s))) \Delta s, \quad t \in I_{\mathbb{T}}:=I \cap \mathbb{T}
$$

onde: $x: I_{\mathbb{T}} \rightarrow \mathbb{R}^{n}$ é a função incógnita; $\mathbb{T}$ é uma escala temporal, isto é, um subconjunto fechado e não-vazio de números reais; $k: I_{\mathbb{T}} \times I_{\mathbb{T}} \times \mathbb{R}^{n} \rightarrow \mathbb{R}^{n}$ e $f: I_{\mathbb{T}} \rightarrow \mathbb{R}^{n}$ são funções dadas; $\sigma$ é uma função definida na próxima seção; e $I$ é um determinado subintervalo de $\mathbb{R}$.

Motivados por Kulik e Tisdell (2008), nós estabelecemos um resultado de existência de soluções para a Eq. (1). Mais especificamente, nós obtemos um análogo do Teorema 5.7 dado por Kulik e Tisdell (2008) para a Eq. (1).

\section{Preliminares}

Nessa seção consideramos conceitos e resultados básicos utilizados no desenvolvimento do presente trabalho.

\subsection{Escalas Temporais}

Definimos a função $\sigma: \mathbb{T} \rightarrow \mathbb{T}$ como

$$
\sigma(t)=\inf \{s \in \mathbb{T}: s>t\}
$$

e a função $\rho: \mathbb{T} \rightarrow \mathbb{T}$ como

$$
\rho(t)=\sup \{s \in \mathbb{T}: s<t\}
$$

Estamos supondo que $\inf \emptyset=\sup \mathbb{T} \operatorname{e} \sup \emptyset=\inf \mathbb{T}$. 
Seja $\mu: \mathbb{T} \rightarrow[0,+\infty)$ dada por

$$
\mu(t)=\sigma(t)-t
$$

Defina também $\mathbb{T}^{\kappa}$ como $\mathbb{T}^{\kappa}=\mathbb{T} \backslash(\rho(\sup \mathbb{T}) \text {, sup } \mathbb{T}]_{\mathbb{T}}$ se $\sup \mathbb{T}<\infty$ e $\mathbb{T}^{\kappa}=\mathbb{T}$ se $\sup \mathbb{T}=\infty$.

Considere uma função $f: \mathbb{T} \rightarrow \mathbb{R}$ e $t \in \mathbb{T}^{\kappa}$. Se existe $\xi \in \mathbb{R}$ tal que, para todo $\varepsilon>0$ existe $\delta>0$ de modo que

$$
|f(\sigma(t))-f(s)-\xi(\sigma(t)-s)| \leq \varepsilon|\sigma(t)-s|
$$

para todo $s \in(t-\delta, t+\delta) \cap \mathbb{T}$, diz-se que $\xi$ é a derivada delta de $f$ em $t$ e denota-se $\xi=f^{\Delta}(t)$.

Dada uma função $f: \mathbb{T} \rightarrow \mathbb{R}^{n}$ e $t \in \mathbb{T}^{\kappa}$, diz-se que $f$ é $\Delta$-diferenciável em $t$ se cada função coordenada $f_{i}: \mathbb{T} \rightarrow \mathbb{R}$ for $\Delta$-diferenciável em $t$. Neste caso $f^{\Delta}(t)=\left(f_{1}^{\Delta}(t), \ldots, f_{n}^{\Delta}(t)\right)$.

A seguir enunciamos alguns resultados básicos do cálculo em escalas temporais. O Teorema 2.1 enunciado abaixo pode ser obtido facilmente do Teorema 1.16 dado em Bohner e Peterson (2001).

Teorema 2.1. Considere uma função $f: \mathbb{T} \rightarrow \mathbb{R}^{n}$ e $t \in \mathbb{T}^{\kappa}$. Então, valem as seguintes propriedades:

(i) Se $f$ é $\Delta$-diferenciável em $t$, então $f$ é contínua em $t$.

(ii) Se $f$ é contínua em $t$ e $\sigma(t)>t$, então $f$ é $\Delta$-diferenciável em $t$. Além disso,

$$
f^{\Delta}(t)=\frac{f(\sigma(t))-f(t)}{\mu(t)} .
$$

(iii) Se $\sigma(t)=t$, então $f$ é $\Delta$-diferenciável em $t$ se, e somente se, o limite

$$
\lim _{s \underset{\mathbb{T}}{\rightarrow} t} \frac{f(t)-f(s)}{t-s}
$$

existe como um elemento de $\mathbb{R}^{n}$. Neste caso,

$$
f^{\Delta}(t)=\lim _{s \underset{\mathbb{T}}{\rightarrow} t} \frac{f(t)-f(s)}{t-s} .
$$

(iv) Se $f$ é $\Delta$-diferenciável em $t$, então

$$
f(\sigma(t))=f(t)+\mu(t) f^{\Delta}(t) .
$$

Definição 2.2. Seja $f: \mathbb{T} \rightarrow \mathbb{R}^{n}$. Se $F^{\Delta}(t)=f(t)$, definimos a integral delta como

$$
\int_{a}^{t} f(s) \Delta s=F(t)-F(a) .
$$


Denotaremos por $C_{r d}\left(\mathbb{T} ; \mathbb{R}^{n}\right)$ o conjunto de todas as funções $f: \mathbb{T} \rightarrow \mathbb{R}^{n}$ rd-contínuas.

Definição 2.3. Uma função $f: \mathbb{T} \rightarrow \mathbb{R}^{n}$ é rd-contínua se $f$ é contínua em cada ponto $t \in \mathbb{T}$ tal que $\sigma(t)=t$ e $\lim _{s \rightarrow t^{-}} f(s)$ existe e é finito em cada ponto $t \in \mathbb{T}$ tal que $\rho(t)=t$.

Como podemos ver em Bohner e Peterson (2001), cada função rd-contínua é delta-integrável.

\subsection{Função Exponencial}

Definimos o conjunto das funções regressivas, $\mathcal{R}$, por

$$
\mathcal{R}:=\left\{p \in C_{r d}(\mathbb{T} ; \mathbb{R}): 1+\mu(t) p(t) \neq 0, \forall t \in \mathbb{T}\right\}
$$

Se $p \in \mathcal{R}$, a única solução para o problema de valor inicial

$$
x^{\Delta}=p(t) x, \quad x(a)=1,
$$

é dada pela função exponencial $e_{p}(t, a)$ na escala temporal $\mathbb{T}$, onde

$$
e_{p}(t, a)=\exp \left(\int_{a}^{t} \xi_{\mu(\tau)}(p(\tau)) \Delta \tau\right)
$$

$\operatorname{com} \xi_{h}(z)=\frac{\log (1+h z)}{h}$ se $h \neq 0$ e $\xi_{h}(z)=z$ se $h=0$, e log é a função logarítmica principal.

\subsection{Equicontinuidade}

Definição 2.4. Seja $E$ uma coleção de funções $f: \mathbb{T} \rightarrow \mathbb{R}^{n}$. Diz-se que $E$ é equicontínuo se para todo $\epsilon>0$ existe $\delta>0$ tal que

$$
\|f(t)-f(s)\|<\epsilon
$$

para toda $f \in E$ e para cada $t, s \in \mathbb{T}$ satisfazendo $|t-s|<\delta$.

Abaixo enunciamos o Teorema de Arzela-Ascoli, que pode ser encontrado em Rudin (1987).

Teorema 2.5. Considere um conjunto compacto $K$ e uma coleção $E$ de funções $f: K \rightarrow \mathbb{R}^{n}$. Suponha que $E$ seja equicontínuo e limitado na norma do supremo. Então toda sequência de $E$ possui subsequência que converge uniformemente em $\mathrm{K}$.

O Teorema do Ponto Fixo de Schäfer se encontra enunciado abaixo e pode ser encontrado em Kulik e Tisdell (2008). 
Teorema 2.6. Seja $X$ um espaço de Banach e seja $F: X \rightarrow X$ compacta. Suponha que o conjunto

$$
\Gamma=\{x \in X: x=\lambda F(x) \text { para algum } \lambda \in[0,1)\}
$$

seja limitado. Então F tem pelo menos um ponto fixo.

\section{Resultados Principais}

O resultado de existência de soluções obtido aqui para a Eq. (1), é afirmado no Teorema 3.3.

Seja $C\left([a, b]_{\mathbb{T}}, \mathbb{R}^{n}\right)$ o conjunto de todas as funções contínuas com domínio $[a, b]_{\mathbb{T}}$ e contradomínio $\mathbb{R}^{n}$.

Considere uma constante $\beta>0$. Defina a métrica $d_{\beta}: C\left([a, b]_{\mathbb{T}}, \mathbb{R}^{n}\right) \times C\left([a, b]_{\mathbb{T}}, \mathbb{R}^{n}\right) \rightarrow[0, \infty)$ como

$$
d_{\beta}(x, y)=\sup _{t \in[a, b]_{\mathbb{T}}} \frac{\|x(t)-y(t)\|}{e_{\beta}(t, a)}
$$

e as normas $\|\cdot\|_{\beta},\|\cdot\|_{0}: C\left([a, b]_{\mathbb{T}}, \mathbb{R}^{n}\right) \rightarrow[0, \infty)$ por

$$
\|x\|_{\beta}=\sup _{t \in[a, b]_{\mathbb{T}}} \frac{\|x(t)\|}{e_{\beta}(t, a)}
$$

e

$$
\|x\|_{0}=\sup _{t \in[a, b]_{\mathbb{T}}}\|x(t)\|
$$

O Lema abaixo pode ser encontrado em Kulik e Tisdell (2008).

Lema 3.1. Se $\beta>0$ é uma constante, então:

1. $d_{\beta}$ é uma métrica;

2. $\left(C\left([a, b]_{\mathbb{T}}, \mathbb{R}^{n}\right), d_{\beta}\right)$ é um espaço métrico completo;

3. $\|x\|_{\beta}$ é uma norma e é equivalente à norma do supremo $\|x\|_{0}$;

4. $\left(C\left([a, b]_{\mathbb{T}}, \mathbb{R}^{n}\right),\|\cdot\|_{\beta}\right)$ é um espaço de Banach.

A seguir consideramos as hipóteses $\mathrm{H} 1$ e $\mathrm{H} 2$ para uma função $k:[a, b]_{\mathbb{T}} \times[a, b]_{\mathbb{T}} \times \mathbb{R}^{n} \rightarrow \mathbb{R}^{n}$.

H1 Existe uma constante $C>0$ satisfazendo

$$
\left\|k\left(t_{1}, s, p\right)-k\left(t_{2}, s, p\right)\right\| \leq C\left|t_{1}-t_{2}\right| .
$$

para quaisquer $\left(t_{1}, t_{2}\right) \in[a, b]_{\mathbb{T}} \times[a, b]_{\mathbb{T}}, s \in[a, b]_{\mathbb{T}}$ e $p \in \mathbb{R}^{n}$. 
$\mathrm{H} 2$ Existam constantes $L>0$ e $N \geq 0$ tais que

$$
\|k(t, s, p)\| \leq L\|p\|+N
$$

para quaisquer $(t, s) \in[a, b]_{\mathbb{T}} \times[a, b]_{\mathbb{T}}$ e $p \in \mathbb{R}^{n}$.

Lema 3.2. Considere o espaço normado $\left(C\left([a, b]_{\mathbb{T}} ; \mathbb{R}^{n}\right),\|\cdot\|_{0}\right)$ e seja $F: C\left([a, b]_{\mathbb{T}} ; \mathbb{R}^{n}\right) \rightarrow C\left([a, b]_{\mathbb{T}} ; \mathbb{R}^{n}\right)$ a função definida por

$$
[F x](t):=f(t)+\int_{a}^{t} k\left(t, s, x(\sigma(s)) \Delta s, \quad t \in[a, b]_{\mathbb{T}} .\right.
$$

Sejam $k:[a, b]_{\mathbb{T}} \times[a, b]_{\mathbb{T}} \times \mathbb{R}^{n} \rightarrow \mathbb{R}^{n}$ e $f:[a, b]_{\mathbb{T}} \rightarrow \mathbb{R}^{n}$ funções contínuas. Se $k$ satisfaz as hipóteses H1 e H2, então F é compacta.

Demonstração. Sejam $\bar{x} \in C\left([a, b]_{\mathbb{T}} ; \mathbb{R}^{n}\right)$ e $\left\{x_{i}\right\} \subset C\left([a, b]_{\mathbb{T}} ; \mathbb{R}^{n}\right)$ tais que $\left\|x_{i}-\bar{x}\right\|_{0} \rightarrow 0$. Para cada $i$, seja $t_{i} \in[a, b]_{\mathbb{T}}$ tal que

$$
\left\|F x_{i}-F \bar{x}\right\|_{0}=\left\|\int_{a}^{t_{i}}\left(k\left(t_{i}, s, x_{i}(\sigma(s))\right)-k\left(t_{i}, s, \bar{x}(\sigma(s))\right)\right) \Delta s\right\| .
$$

Logo, existe uma subsequência de $\left\{t_{i}\right\}$, não reindexamos, tal que $t_{i} \rightarrow t$, para algum $t \in[a, b]_{\mathbb{T}}$. Assim,

$$
\begin{aligned}
\left\|F x_{i}-F \bar{x}\right\|_{0} \leq & \int_{a}^{t_{i}}\left\|k\left(t_{i}, s, x_{i}(\sigma(s))\right)-k\left(t_{i}, s, \bar{x}(\sigma(s))\right)\right\| \Delta s \\
\leq & \int_{a}^{b}\left\|k\left(t_{i}, s, x_{i}(\sigma(s))\right)-k\left(t_{i}, s, \bar{x}(\sigma(s))\right)\right\| \Delta s \\
\leq & \int_{a}^{b}\left\|k\left(t_{i}, s, x_{i}(\sigma(s))\right)-k(t, s, \bar{x}(\sigma(s)))\right\| \Delta s \\
& +\int_{a}^{b}\left\|k(t, s, \bar{x}(\sigma(s)))-k\left(t_{i}, s, \bar{x}(\sigma(s))\right)\right\| \Delta s
\end{aligned}
$$

e do Teorema da Convergência Dominada, considerado por exemplo em Rudin (1987), concluímos que $\left\|F x_{i}-F \bar{x}\right\|_{0} \rightarrow 0$. Logo, $F$ é contínua.

A seguir verificamos que $F$ leva conjuntos limitados em conjuntos relativamente compactos. Para isso, usaremos o Teorema de Arzela-Ascoli. Assim, dada a sequência $\left\{x_{i}\right\}$ com $\left\|x_{i}\right\|_{0} \leq r$, mostremos que a sequência $v_{i}:=F\left(x_{i}\right)$ é limitada e equicontínua.

Seja

$$
M:=\sup \left\{\|k(t, s, p)\|:(t, s) \in[a, b]_{\mathbb{T}} \times[a, b]_{\mathbb{T}},\|p\| \leq r\right\}<\infty .
$$


Temos

$$
\begin{aligned}
\left\|v_{i}\right\|_{0} & =\sup _{t \in[a, b]_{\mathbb{T}}}\left\|v_{i}(t)\right\| \\
& \leq \sup _{t \in[a, b]_{\mathbb{T}}}\|f(t)\|+\sup _{t \in[a, b]_{\mathbb{T}}} \int_{a}^{t} \| k\left(t, s, x_{i}(\sigma(s)) \| \Delta s\right. \\
& \leq \sup _{t \in[a, b]_{\mathbb{T}}}\|f(t)\|+(b-a) M
\end{aligned}
$$

e assim $\left\{v_{i}\right\}$ é limitada.

Se $t_{1}, t_{2} \in[a, b]_{\mathbb{T}}$ e $t_{1}<t_{2}$ segue que

$$
\begin{aligned}
& \left\|v_{i}\left(t_{1}\right)-v_{i}\left(t_{2}\right)\right\| \\
& \leq\left\|f\left(t_{1}\right)-f\left(t_{2}\right)\right\| \\
& \quad+\int_{a}^{t_{1}}\left\|k\left(t_{1}, s, x_{i}(\sigma(s))\right)-k\left(t_{2}, s, x_{i}(\sigma(s))\right)\right\| \Delta s+\int_{t_{1}}^{t_{2}}\left\|k\left(t_{2}, s, x_{i}(\sigma(s))\right)\right\| \Delta s \\
& \leq\left\|f\left(t_{1}\right)-f\left(t_{2}\right)\right\|+C(b-a)\left|t_{1}-t_{2}\right|+\left(L\left\|x_{i}\right\|_{0}+N\right)\left|t_{1}-t_{2}\right| \\
& \leq\left\|f\left(t_{1}\right)-f\left(t_{2}\right)\right\|+C(b-a)\left|t_{1}-t_{2}\right|+(L r+N)\left|t_{1}-t_{2}\right| .
\end{aligned}
$$

De modo análogo, se $t_{1}>t_{2}$ temos que

$$
\begin{aligned}
& \left\|v_{i}\left(t_{1}\right)-v_{i}\left(t_{2}\right)\right\| \\
& \leq\left\|f\left(t_{1}\right)-f\left(t_{2}\right)\right\|+C(b-a)\left|t_{1}-t_{2}\right|+\left(L\left\|x_{i}\right\|_{0}+N\right)\left|t_{1}-t_{2}\right| \\
& \leq\left\|f\left(t_{1}\right)-f\left(t_{2}\right)\right\|+C(b-a)\left|t_{1}-t_{2}\right|+(L r+N)\left|t_{1}-t_{2}\right|
\end{aligned}
$$

e como $f$ é uniformemente contínua concluímos que $\left\{v_{i}\right\}$ é equicontínua. O Lema segue agora do Teorema de Arzela-Ascoli.

Teorema 3.3. Considere a equação integral dada na Eq. (1) com $I_{\mathbb{T}}:=[a, b]_{\mathbb{T}}$. Sejam $k: I_{\mathbb{T}} \times I_{\mathbb{T}} \times$ $\mathbb{R}^{n} \rightarrow \mathbb{R}^{n}$ e $f: I_{\mathbb{T}} \rightarrow \mathbb{R}^{n}$ funções contínuas. Suponha que a função $k$ satisfaz as hipóteses $H 1$ e $H 2$. Suponha também que $e_{L}(b, a)<2$ e $L\|\mu\|_{0}<1$. Então a Eq. (1) tem pelo menos uma solução.

Demonstração. Para a obtenção de pelo menos uma solução para a Eq. (1), usaremos o Teorema do Ponto Fixo de Schäfer. Para isso, considere o espaço normado $\left(C\left([a, b]_{\mathbb{T}} ; \mathbb{R}^{n}\right),\|\cdot\|_{L}\right)$ e a família de equações

$$
x=\lambda F x, \quad \lambda \in[0,1)
$$

onde $F: C\left([a, b]_{\mathbb{T}} ; \mathbb{R}^{n}\right) \rightarrow C\left([a, b]_{\mathbb{T}} ; \mathbb{R}^{n}\right)$ é definida na Eq. (2). Como $\|\cdot\|_{L} \mathrm{e}\|\cdot\|_{0}$ são equivalentes, do Lema 3.2 temos que $F$ é compacta. 
Seja $\lambda \in[0,1)$ tal que $x \in C\left([a, b]_{\mathbb{T}} ; \mathbb{R}^{n}\right)$ é uma solução da Eq. (3). Temos que

$$
\begin{aligned}
\|x\|_{L} & =\|\lambda F x\|_{L} \\
& \leq\|f\|_{L}+\sup _{t \in[a, b]_{\mathbb{T}}} \frac{1}{e_{L}(t, a)} \int_{a}^{t} \| k(t, s, x(\sigma(s)) \| \Delta s \\
& \leq\|f\|_{L}+\sup _{t \in[a, b]_{\mathbb{T}}} \frac{1}{e_{L}(t, a)} \int_{a}^{t}(L\|x(\sigma(s))\|+N) \Delta s \\
& =\|f\|_{L}+\sup _{t \in[a, b]_{\mathbb{T}}} \frac{1}{e_{L}(t, a)}\left[\int_{a}^{t} L e_{L}(\sigma(s), a) \frac{\|x(\sigma(s))\|}{e_{L}(\sigma(s), a)} \Delta s+N(t-a)\right] \\
& \leq\|f\|_{L}+\sup _{t \in[a, b]_{\mathbb{T}}} \frac{1}{e_{L}(t, a)} \int_{a}^{t} L e_{L}(\sigma(s), a)\|x\|_{L} \Delta s+\sup _{t \in[a, b]_{\mathbb{T}}} \frac{N(t-a)}{e_{L}(t, a)} \\
& =\|f\|_{L}+\sup _{t \in[a, b]_{\mathbb{T}}} \frac{1}{e_{L}(t, a)} \int_{a}^{t} L\|x\|_{L}[1+\mu(s) L] e_{L}(s, a) \Delta s+N(b-a) \\
& \leq\|f\|_{L}+\sup _{t \in[a, b]_{\mathbb{T}}} \frac{1}{e_{L}(t, a)} \int_{a}^{t} L\|x\|_{L}\left[1+\|\mu\|_{0} L\right] e_{L}(s, a) \Delta s+N(b-a) \\
& =\|f\|_{L}+\sup _{t \in[a, b]_{\mathbb{T}}} \frac{1}{e_{L}(t, a)}\left[1+\|\mu\|_{0} L\right]\|x\|_{L}\left(e_{L}(t, a)-1\right)+N(b-a) \\
& =\|f\|_{L}+\left[1+\|\mu\|_{0} L\right]\|x\|_{L}\left(1-\frac{1}{e_{L}(b, a)}\right)+N(b-a) \\
& \leq\|f\|_{L}+2\|x\|_{L}\left(1-\frac{1}{e_{L}(b, a)}\right)+N(b-a)
\end{aligned}
$$

e então

$$
\|x\|_{L} \leq \frac{e_{L}(b, a)\left[\|f\|_{L}+N(b-a)\right]}{2-e_{L}(b, a)}
$$

Assim, do Teorema do Ponto Fixo de Schäfer concluímos que $F$ tem pelo menos um ponto fixo. Portanto a Eq. (1) tem pelo menos uma solução.

\section{Conclusões}

O presente artigo contribui para a teoria de escalas temporais. Mais especificamente, o artigo estabelece um resultado de existência de soluções para equações integrais de Volterra em escalas temporais. Assim, usando o Teorema do Ponto Fixo de Schäfer, a existência de soluções para equações integrais de Volterra em escalas temporais é estabelecida. O resultado obtido aqui se soma à literatura de existência de soluções para equações integrais de Volterra em escalas temporais. 


\section{Referências}

ATICI, F. M.; BILES, D. C. First order dynamic inclusions on time scales. Journal of Mathematical Analysis and Applications, v. 292, n. 1, p. 222-237, 2004.

BOHNER, M.; PETERSON, A. Advances in dynamic equations on time scales. Boston, MA: Birkhäuser, 2003.

BOHNER, M.; PETERSON, A. Dynamic equations on time scales: an introduction with applications. Boston: Birkhäuser Boston, Inc., 2001.

FRIGON, M.; GILBERT, H. Systems of first order inclusions on time scales. Topological Methods in Nonlinear Analysis, v. 37, n. 1, p. 147-163, 2011.

HILGER, S. Ein Masskettenkalkül mit Anwendung auf Zentrumsmanningfaltigkeiten. $\mathrm{PhD}$ thesis, Universität Würzburg, 1988.

KARPUZ, B. Volterra theory on time scales. Results in Mathematics, v. 65, n. 3-4, p. 263-292, 2014.

KULIK, T.; TISDELL, C. C. Volterra integral equations on time scales: basic qualitative and quantitative results with applications to initial value problems on unbounded domains.

International Journal of Difference Equations, v. 3, n. 1, p. 103-133, 2008.

MESSINA, E.; RUSSO, E.; VECCHIO, A. Volterra integral equations on time scales: stability under constant perturbations via Liapunov direct method. Ricerche di Matematica, v. 64, n. 2, p. 345-355, 2015.

MESSINA, E.; VECCHIO, A. Stability and convergence of solutions to Volterra integral equations on time scales. Discrete Dynamics in Nature and Society, Art. ID 612156, 6 p., 2015.

RUDIN, W. Real and complex analysis. New York: McGraw-Hill Book Co., 1987.

SANTOS, I. L. D.; SILVA, G. N. Absolute continuity and existence of solutions to dynamic inclusions in time scales. Mathematische Annalen, v. 356, n. 1, p. 373-399, 2013.

SANTOS, I. L. D. dos. On qualitative and quantitative results for solutions to first-order dynamic equations on time scales. Boletín de la Sociedad Matemática Mexicana. Third Series, v. 21, n. 2, p. 205-218, 2015.

SANTOS, I. L. D. dos. On Volterra integral equations on time scales. Mediterranean Journal of Mathematics, v. 12, n. 2, p. 471-480, 2015. 